\title{
Measurement of the Secondary Recrystallization in Copper by X-ray Diffraction and ECP*
}

\author{
By Harumitsu Makita**, Shuji Hanada*** and Osamu Izumi***
}

\begin{abstract}
In order to clarify the process of the secondary recrystallization in commercial copper, textures, microstructures and individual grain orientations have been examined by means of X-ray diffraction, scanning electron microscopy (SEM) and SEM-electron channeling pattern (SEM-ECP) methods.

$\mathrm{X}$-ray diffraction showed that the primary recrystallization texture was dependent on initial grain size before cold rolling. A fine grained sample showed the texture with a very sharp cube orientation, while a coarse grained sample showed a diffuse cube orientation mixed with a random one. SEM-ECP observations revealed that some grains with orientations identical with those observed in the secondaryrecrystallized grains were already present in the primary-recrystallized grains with the sharp cube orientation. On the other hand, the banded structure elongated along rolling direction, consisting of alternately clustered primary grains with a very sharp cube and with a diffuse cube orientation, was observed in the primary-recrystallized sample with the diffuse cube orientation. Orientations of secondary-recrystallized grains were found to correspond to those in clusters of the diffuse cube-oriented grains.

In addition to initial grain size, rolling procedure, annealing conditions and the ear produced by heavy rolling reduction were observed to affect secondary recrystallization.

The orientation relationships of secondary-recrystallized grains to the primary cube texture are discussed on the basis of the Coincidence Site Lattice model.
\end{abstract}

(Received January 26, 1987)

Keywords: copper, primary recrystallization, secondary recrystallization, texture, Coincidence Site Lattice model

\section{Introduction}

It is well known that the primary recrystallization texture of heavily rolled copper sheet shows cube texture, although it is affected by various factors such as deformation procedure, annealing condition, initial grain size, rolling temperature and chemical composition $^{(1)-(8)}$. However, secondary recrystallization texture of the sheet which showed originally cube texture in primary recrystallization has not been well established. For example, Dahl and Pawlek ${ }^{(9)}$ and Cook and Richards ${ }^{(10)}$ reported $\{210\}\langle 001\rangle$ and $\{110\}\langle 112\rangle$ as the preferred orientation of secondary recrystal-

* A part of this paper was originally published in Japanese in J. Japan Inst. Metals, 49 (1985), 1027.

** Kawai Musical Instruments Mfg. Co., Ltd.,: Hamamatsu. Present address: The Research Institute for Iron, Steel and Other Metals, Tohoku University, Sendai.

*** The Research Institute for Iron, Steel and Other Metals, Tohoku University, Sendai. lization, respectively. Kronberg and Wilson ${ }^{(11)}$ and Sharp and Dunn ${ }^{(12)}$ observed the $19^{\circ}(0.33$ $\mathrm{rad})\langle 100\rangle$ and $22^{\circ}(0.38 \mathrm{rad})$ or $38^{\circ}(0.66 \mathrm{rad})$ $\langle 111\rangle$ orientation relationships between the cube texture and the secondary recrystallization orientations. Abe and Yamada ${ }^{(13)}$ found that the cube texture develops as the secondary recrystallization texture in a high purity electrolytic nickel sheet.

On the other hand, extensive studies of secondary recrystallization in a silicon steel sheet have recently revealed that the potential nuclei of secondary (Goss) grains exist in the primary grains at a certain depth under the sheet surface ${ }^{(14)-(17)}$ and such grains sometimes do not have size advantage ${ }^{(16)(17)}$, although the largegrain hypothesis for secondary recrystallization nucleation has been widely accepted ${ }^{(18)-(20)}$. Very little information has been available on the potential nuclei of secondary grains in primary grains of copper sheet. Accordingly, it is important to examine the orientation of individual grains in a very sharp cubic structure for a better understanding of secondary recrys- 
tallization not only in copper but also in silicon steel sheet. In order to clarify the secondary recrystallization in copper, the textures after primary and secondary recrystallization were measured by means of X-ray diffraction and the selected area channeling pattern method in a scanning electron microscope.

\section{Experimental Procedure}

The starting material used throughout the investigation was a commercial copper (OFHC) bar, containing 0.0009 mass $\% \mathrm{O}$, 0.0003 mass \% P and 0.01 mass\% S. Initial grain size was approximately $0.05 \mathrm{~mm}$ (a fine grained sample). A block with the dimensions of $50 \mathrm{~mm} \times 40 \mathrm{~mm} \times 35 \mathrm{~mm}$ was cut from the bar and straight-rolled to sheet about $0.35 \mathrm{~mm}$ in thickness. This sample will be named A.

In order to investigate the effect of initial grain size on recrystallization, coarse grained material was prepared from the commercial copper bar by $30 \%$ compression followed by annealing at $1273 \mathrm{~K}$ for $10.8 \mathrm{ks}$ in a vacuum. The grains grew to aprroximately $0.5 \mathrm{~mm}$ (a coarse grained sample) and this material was straight-rolled to sheet about $0.35 \mathrm{~mm}$ in thickness. This sample will be named $B$. The amount of reduction per every pass was $1 \mathrm{~mm}$ using the work rolls $250 \mathrm{~mm}$ in diameter. The reduction in thickness of all the samples used in this experiment was approximately $99 \%$.

During heavy cold rolling, the ear was produced approximately $2 \sim 3 \mathrm{~mm}$ in width at both edges of the sheet. This ear region was said to affect the secondary recrystallization ${ }^{(11)}$, since it undergoes a different metal flow in comparison with the central portion of the sheet. Accordingly, we prepared the samples with and without the ear for comparison. Furthermore, the edge regions after cutting-off the ear were etched with $\mathrm{HNO}_{3}$ to remove extra strain introduced by cutting. Annealing for recrystallization, unless stated otherwise, was carried out for a constant time of $7.2 \mathrm{ks}$. The textures were measured by a Schulz reflection method. The $\alpha$ angle was measured between $20^{\circ}(0.39$ $\mathrm{rad})$ and $90^{\circ}(1.57 \mathrm{rad})$. The annealed specimens with dimensions of approximately $35 \mathrm{~mm} \times 20 \mathrm{~mm} \times 0.35 \mathrm{~mm}$ were etched with
$\mathrm{FeCl}_{3}+\mathrm{HCl}+\mathrm{H}_{2} \mathrm{O}$ mixture. The microstructures were observed by an optical microscope (OM) and a scanning electron microscope (SEM). In addition, the selected area channeling pattern method in a scanning electron microscope (SEM-ECP) was also used to determine an individual grain orientation.

\section{Results}

Systematic study on secondary recrystallization in copper was performed by Kronberg and Wilson ${ }^{(11)}$. All of their cold rolled sheets were annealed in a relatively low vacuum. Therefore, at the beginning we reexamined recrystallization of copper in a low vacuum of $2 \mathrm{~Pa}$ for comparison with their results. A cube texture due to primary recrystallization was observed in the sample A annealed at temperatures above $473 \mathrm{~K}$, where the cube texture became sharp with increasing annealing temperature. Secondary recrystallization did not occur in the sample A without ear even during annealing at temperatures above $1273 \mathrm{~K}$ in agreement with their observation ${ }^{(11)}$. A typical example of the sharp cube texture is shown in Fig. 1, where the sample was annealed at 1333 $\mathrm{K}$ for $3.6 \mathrm{Ks}$. In order to investigate an orientation distribution of individual grains in the primary-recrystallized sample in more detail, the sample used in X-ray diffraction of Fig. 1 was chemically etched to reveal grain boundaries clearly and observed by SEM and SEMECP. All the grains and their grain boundaries were homogeneously etched. All of 150 grains measured were within $6^{\circ}(0.10 \mathrm{rad})$ from the ideal cube orientation, and no off-cube oriented grains, which deviated by more than $10^{\circ}(0.17 \mathrm{rad})$ from the cube one, were found by numerous experiments. In addition, no twin traces could be seen in OM. Therefore, the peaks other than the cube orientation shown in Fig. 1 are thought to be ghost.

Kronberg and Wilson ${ }^{(11)}$ showed that secondary recrystallization occurred during annealing of heavily rolled copper sheets when the ear of sheet produced by rolling was not removed. Their result that the presence of ear was important for secondary recrystallization was confirmed in the present experiment. 


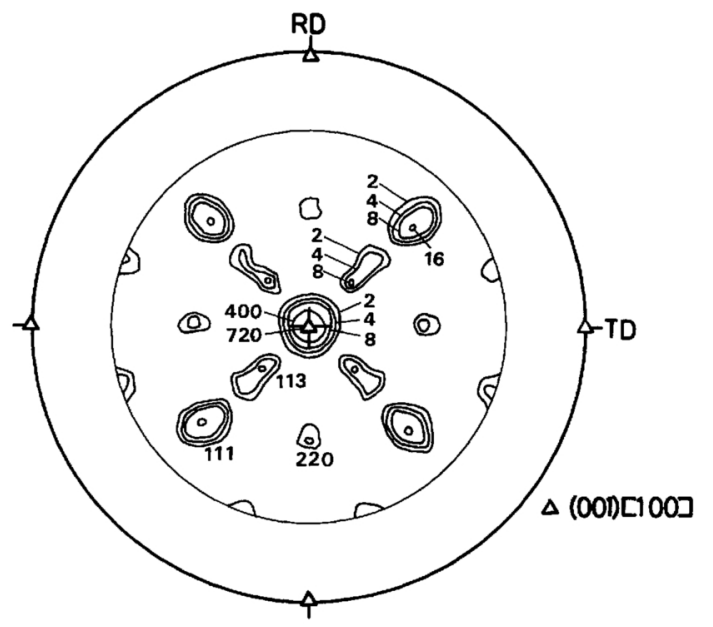

Fig. $1\{100\}$ pole figure of copper sheet after straightrolled by $99 \%$ and annealed for $3.6 \mathrm{ks}$ at $1333 \mathrm{~K}$ in a vacuum of $2 \mathrm{~Pa}$ without ear. Miller indices denote those of ghost peaks.

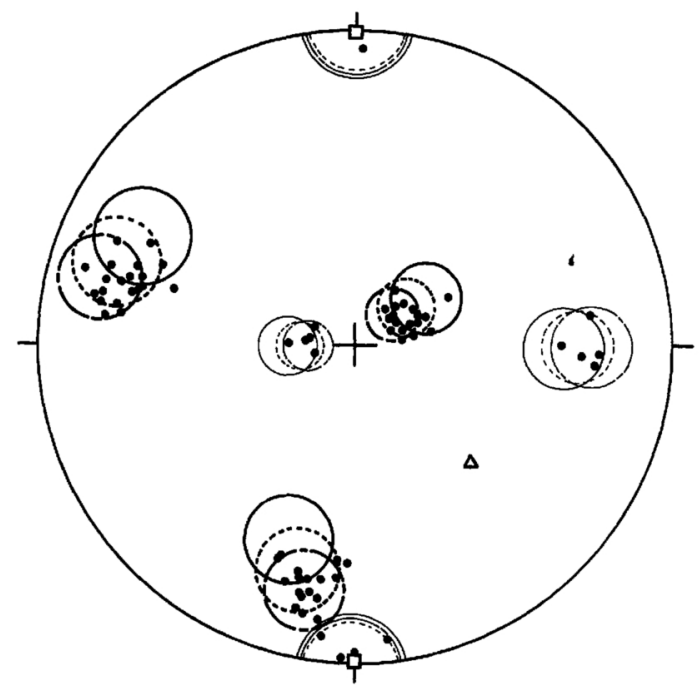

$$
\begin{array}{cc}
-\Sigma 7 \pm(\Delta \theta+6)^{\circ} & -\Sigma 13 a \pm(\Delta \theta+6)^{\circ} \\
\cdots \Sigma 13 b \pm(\Delta \theta+6)^{\circ} & \cdots \Sigma 37 a \pm(\Delta \theta+6)^{\circ} \\
-\Sigma 21 a \pm(\Delta \theta+6)^{\circ} & \cdots \Sigma 25 a \pm(\Delta \theta+6)^{\circ}
\end{array}
$$

Fig. 2 The positions of [100] poles obtained from the secondary-recrystallized grains in copper sheet after straight-rolled by $99 \%$ and annealed for $3.6 \mathrm{ks}$ at $1333 \mathrm{~K}$ in a vacuum of $2 \mathrm{~Pa}$ with ear. Centers of $\pm(\Delta \theta+6)^{\circ}$ circles are at indicated orientations of the center of projection about common [111] and [100] with cube.

Orientations of secondary grains in the sample A with ear are plotted in Fig. 2 after bringing their [111] and [100] poles into the same quadrant. (Various circles will be discussed later.) It is apparent that the secondary grains show preferred orientations. Since secondary recrystallization of this sample was likely to be initiated at the ear, orientations of primary grains in the ear were analysed. The orientations were widely dispersed, but several grains were found to have the same orientations as those of secondary grains in Fig. 2. Orientations of these grains are plotted in Fig. 3.

Various factors other than ear, such as rolling procedure $e^{(8)}$, initial grain size ${ }^{(3)(11)}$, annealing atmosphere ${ }^{(21)}$ etc., have been considered to affect secondary recrystallization. Therefore, the factors were checked in this work. First, we investigate the effect of rolling procedure using the sheet prepared by zigzag-rolling of fine grained copper, where the feed direction was changed approximately $\pm 10^{\circ} \quad(0.17$ rad) to the longitudinal one per every pass. The final thickness of sample was the same as that of A. This sample is named C. The primary

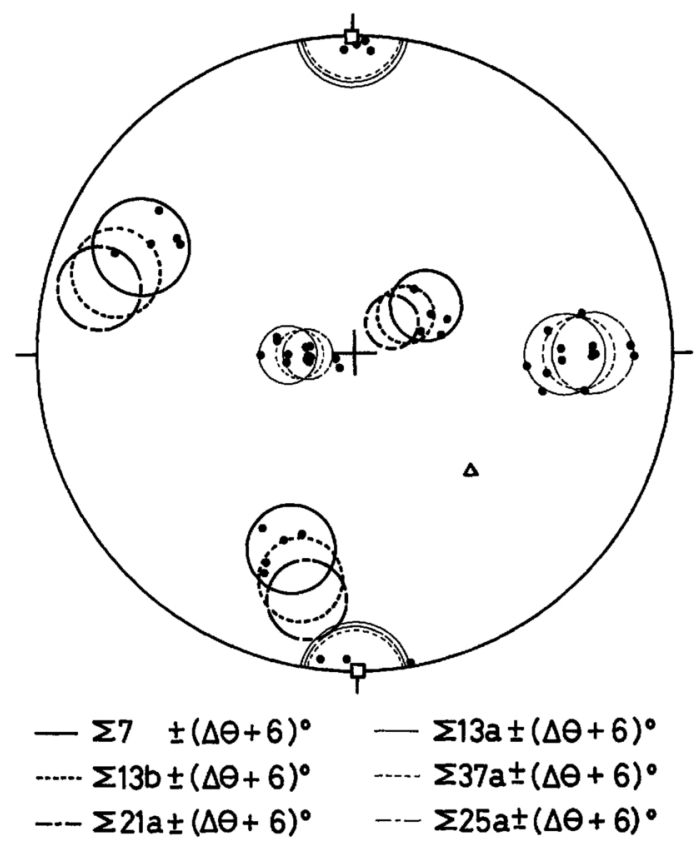

Fig. 3 The positions of [100] poles obtained from the grains in the ear of copper sheet after straight-rolled by $99 \%$ and annealed for $7.2 \mathrm{ks}$ at $1123 \mathrm{~K}$ in a vacuum of $2 \mathrm{~Pa}$. Numerous observations showed no preferred orientation, but several orientations were plotted in this figure. Centers of $\pm(\Delta \theta+6)^{\circ}$ circles are at indicated orientations of the center of projection about common [111] and [100] with cube. 


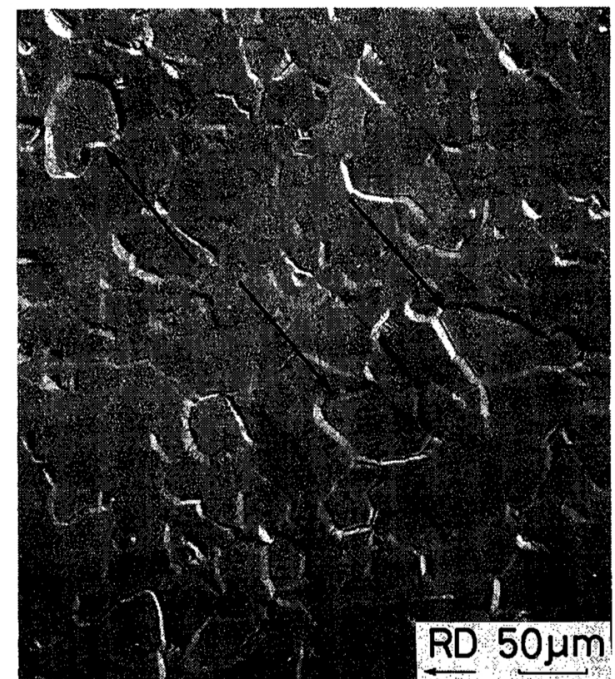

Fig. 4 Scanning electron micrograph of copper sheet after zigzag-rolled by $99 \%$ and annealed for $7.2 \mathrm{ks}$ at $1123 \mathrm{~K}$ in a vacuum of $2 \mathrm{~Pa}$. SEM-ECP observations revealed that the grains indicated by arrows had orientations over $10^{\circ}$ away from cube orientation, while all the other grains showed orientations within $6^{\circ}$ from cube.

recrystallization texture of the sample $\mathrm{C}$ measured by X-ray diffraction showed the same $\{100\}$ pole figure as that shown in Fig. 1. However, an outstanding difference was found by SEM observations, Fig. 4. Grains indicated by arrows are deeply etched in preference to the surrounding grains, although the deeply etched grains were less than $1 \%$ of all the primary grains. These grains were never observed in the primary grains in the sample $A$. SEM-ECP observations revealed that these grains had orientations over $10^{\circ}(0.17 \mathrm{rad})$ away from cube orientation. The orientation distribution of the grains is shown in Fig. 5, indicating a preferred orientation. On the other hand, orientations of grains other than the deeply etched ones were found to be within $6^{\circ}$ $(0.10 \mathrm{rad})$ from the cube orientation in agreement with those of the primary grains of the sample A. Secondary recrystallization occurred in the sample $C$ without ear. Orientations of the secondary grains are plotted in Fig. 6, indicating that the preferred orientation is similar to Fig. 5.

Second, the effect of initial grain size before rolling on secondary recrystallization was investigated using the coarse grained sample $B$.

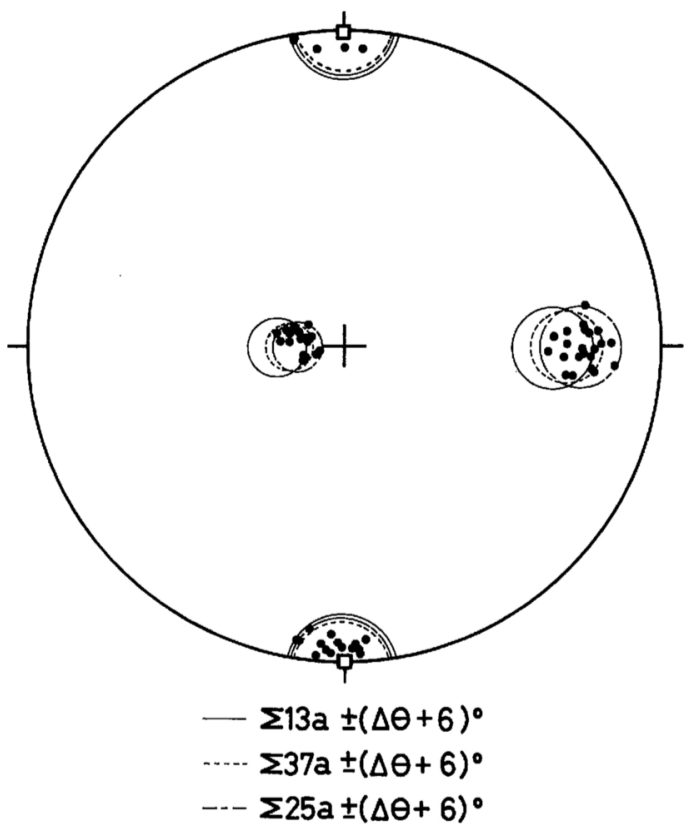

Fig. 5 The positions of [100] poles obtained from the offcube oriented grains. (An example is indicated by arrows in Fig. 4.) Centers of $\pm(\Delta \theta+6)^{\circ}$ circles are at indicated orientations of the center of projection about common [100] with cube.

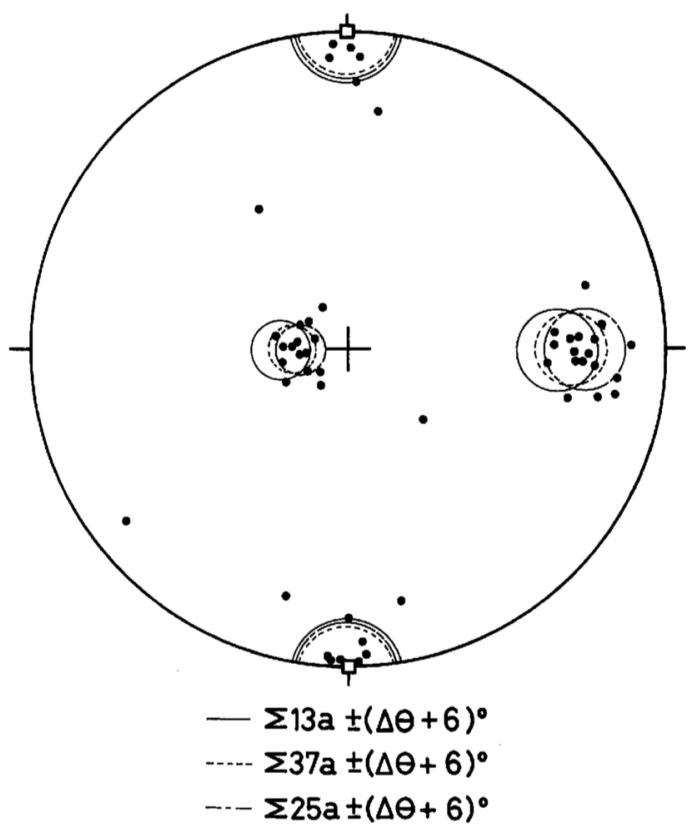

Fig. 6 The positions of [100] poles obtained from the secondary-recrystallized grains in copper after zigzag-rolled by $99 \%$ and annealed for $3.6 \mathrm{ks}$ at $1313 \mathrm{~K}$ in a vacuum of $2 \mathrm{~Pa}$ without ear. Centers of $\pm(\Delta \theta+6)^{\circ}$ circles are at indicated orientations of the center of projection about common [100] with cube. 
Figure 7 shows a $\{100\}$ pole figure of the sample $B$ annealed at $773 \mathrm{~K}$, indicating the scattering from the cube texture in comparison with Fig. 1. No significant change in the scattering was seen in the primary recrystallization texture of the sample $B$ at various temperatures. Figure 8 shows an optical micrograph of the primary-recrystallized sample $\mathrm{B}$. The micro-

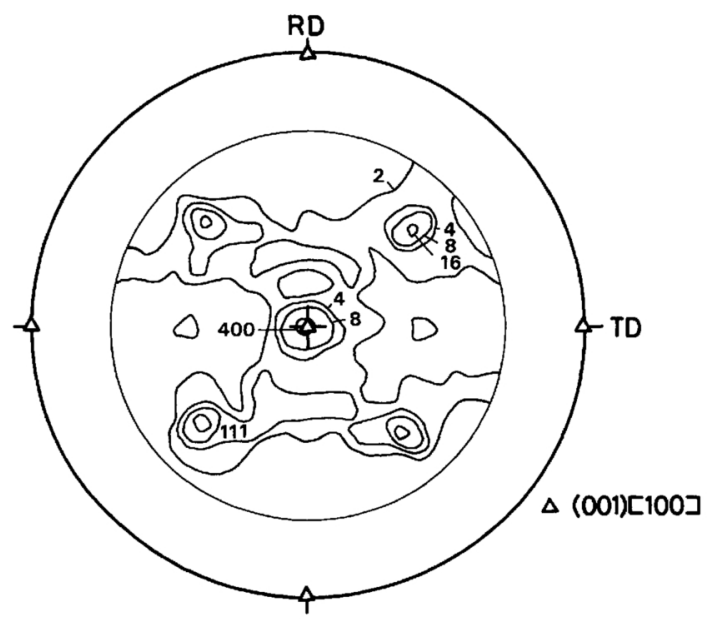

Fig. $7\{100\}$ pole figure of copper sheet after straightrolled by $99 \%$ with coarse grained sample and annealed for $7.2 \mathrm{ks}$ at $773 \mathrm{~K}$ in a vacuum of $2 \mathrm{~Pa}$. The four peaks with intensity level 16 are thought to be ghost ones.

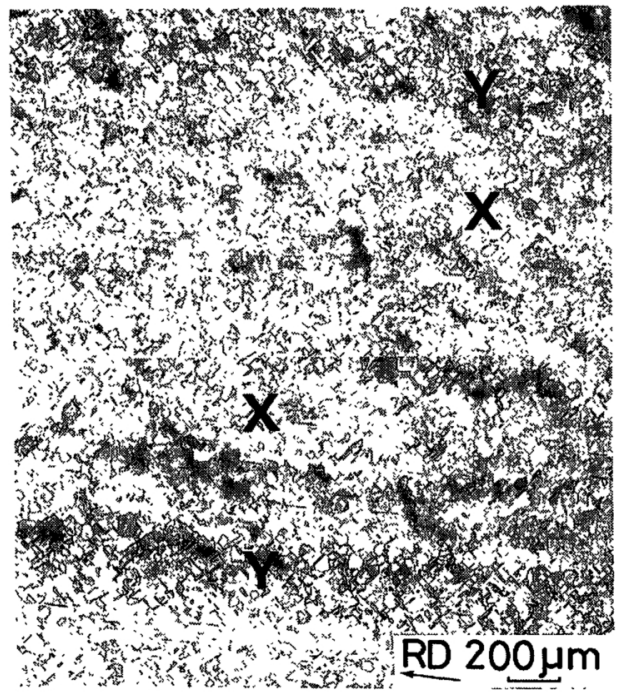

Fig. 8 Optical micrograph of copper sheet after straightrolled by $99 \%$ with coarse grained sample and annealed for $7.2 \mathrm{ks}$ at $773 \mathrm{~K}$ in a vacuum of $2 \mathrm{~Pa}$. The characteristic microstructure, indicated by $\mathrm{X}$ or $\mathrm{Y}$, was observed to extend to the rolling direction. structure consists of grain clusters with smoothly etched and equiaxed grains with a small amount of fine twin traces (regions indicated by $\mathrm{X}$ ), and of grain clusters with welldefined grain boundaries with a lot of twin traces (regions indicated by $\mathrm{Y}$ ). The grain clusters are extended to the rolling direction and the characteristic microstructures may result from inhomogeneous deformation at and within initial grain boundary. SEM-ECP observations on these regions showed that grains in the region $\mathrm{X}$ were of the cube orientation, while stereographic projections of $\{100\}$ poles obtained from the region $Y$ indicated the cube orientation mixed with off-cube ones in correspondence with the result of Fig. 7. The orientations of the off-cube oriented grains showed no preferred orientations. However, orientations of some grains seem to correspond to those in Fig. 2, as plotted in Fig. 9. In the sample B without ear, secondary grains

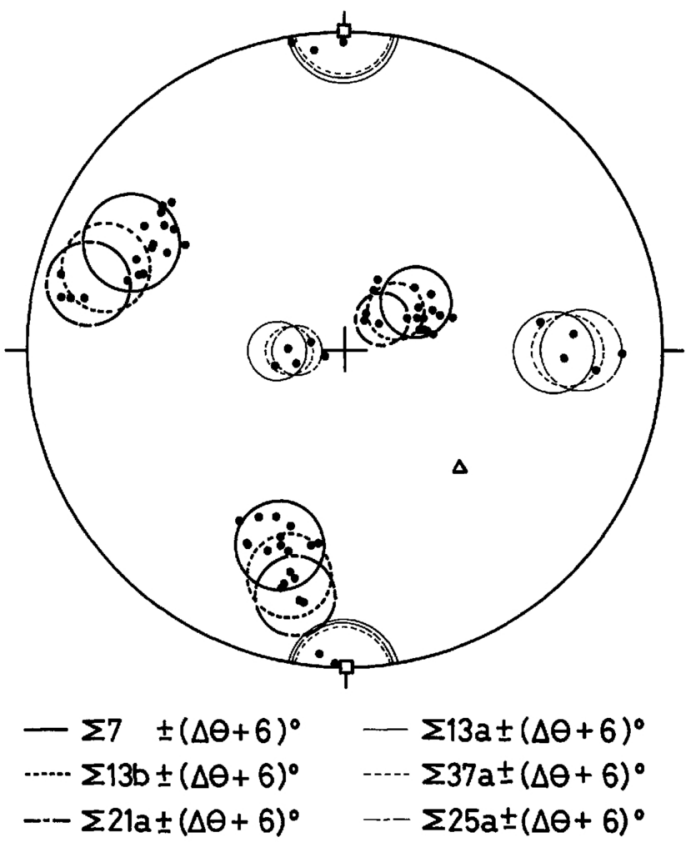

Fig. 9 The positions of [100] poles obtained from the offcube oriented grains in copper sheet after straight-rolled by $99 \%$ with coarse grained sample and annealed for $7.2 \mathrm{ks}$ at $773 \mathrm{~K}$ in a vacuum of $2 \mathrm{~Pa}$. Numerous experiments showed no preferred orientation, but several orientations were plotted in this figure. Centers of $\pm(\Delta \theta+6)^{\circ}$ circles are at indicated orientations of the center of projection about common [111] and [100] with cube. 
began to appear after annealing above $923 \mathrm{~K}$ and arranged in a row parallel to the rolling direction. This characteristic feature seems to be related to the grain clusters observed in the primary recrystallization. Annealing at $1023 \mathrm{~K}$ of the sample B induced complete secondary recrystallization. The orientations of the secondary grains observed by SEM-ECP are plotted in Fig. 10, indicating a slightly pronounced preferred orientation in comparison with Fig. 9.

Annealing experiments in the above-described results were carried out in a vacuum of 2Pa, following Kronberg and Wilson. Third, the effect of annealing atmosphere was checked by comparing the above results with those of the sample annealed in a vacuum of $2 \times 10^{-2} \mathrm{~Pa}$. Secondary recrystallization appeared when the sample $A$ without ear was annealed at $973 \mathrm{~K}$ for $7.2 \mathrm{ks}$ in a vacuum of $2 \times 10^{-2} \mathrm{~Pa}$. This is in contrast to the result of the sample $\mathrm{A}$ without ear annealed at $1333 \mathrm{~K}$ for $3.6 \mathrm{ks}$ in $2 \mathrm{~Pa}$, where secondary recrystalliza-

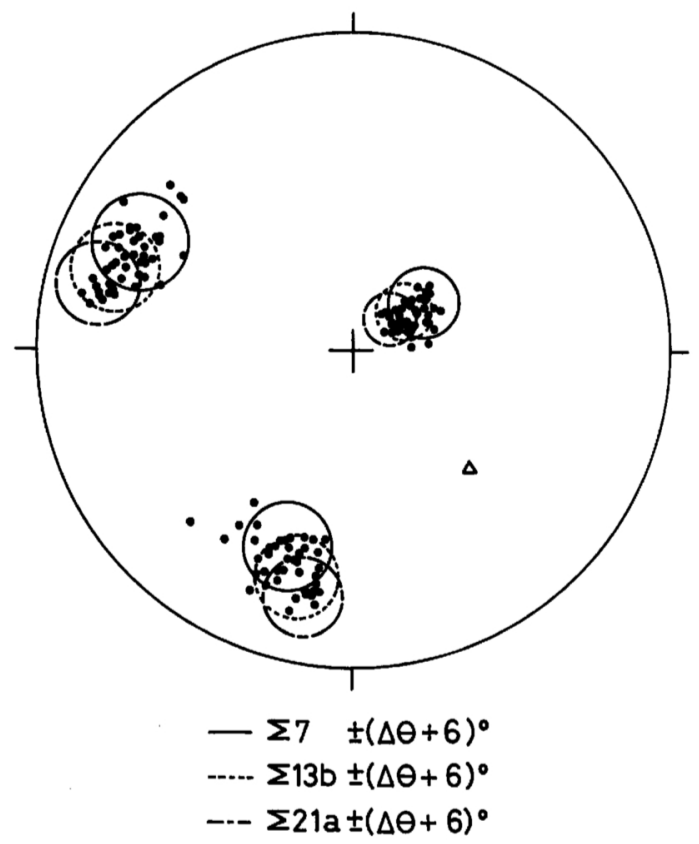

Fig. 10 The positions of [100] poles obtained from the secondary-recrystallized grains in copper sheet after straight-rolled by $99 \%$ with coarse grained sample and annealed for $3.6 \mathrm{ks}$ at $1273 \mathrm{~K}$ in a vacuum of $2 \mathrm{~Pa}$. Centers of $\pm(\Delta \theta+6)^{\circ}$ circles are at indicated orientations of the center of projection about common [111] with cube.

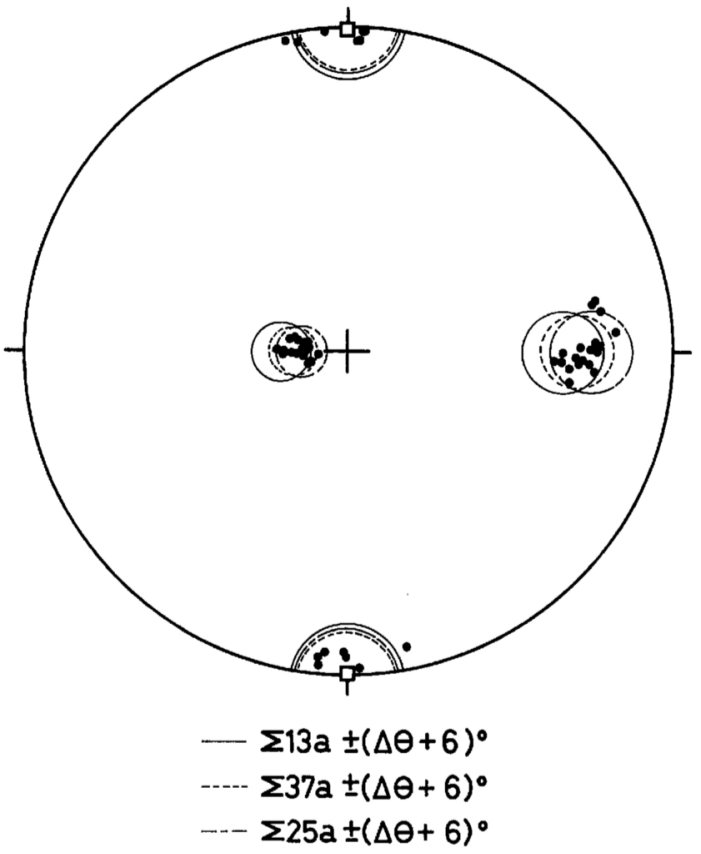

Fig. 11 The positions of [100] poles obtained from the secondary-recrystallized grains in copper sheet after straight-rolled by $99 \%$ and annealed for $3.6 \mathrm{ks}$ at $1313 \mathrm{~K}$ in a vacuum of $2 \times 10^{-2} \mathrm{~Pa}$ without ear. Centers of $\pm(\Delta \theta+6)^{\circ}$ circles are at indicated orientations of the center of projection about common [100] with cube.

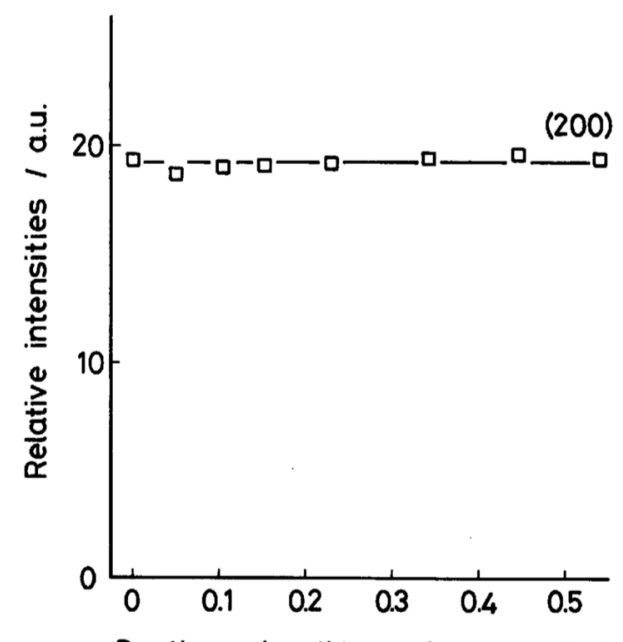

Depth under the surface / $\Delta T / T$

Fig. 12 The variation of intensities of (200) planes through the thickness from the surface to the center of the primary-recrystallized copper sheet after straightrolled by $99 \%$ and annealed for $7.2 \mathrm{ks}$ at $773 \mathrm{~K}$ in a vacuum of $2 \times 10^{-2} \mathrm{~Pa}$. 
tion was never detected. The orientations of secondary grains are plotted in Fig. 11, which is similar to Fig. 6.

Finally, texture was examined as a function of distance from sheet surface, since it was known that texture gradient was observed in silicon steel sheet. That is, the potential nuclei of secondary grains have a tendency to form cluster at $1 / 5 \sim 1 / 4^{(14)}, 1 / 10^{(15)}$ or $1 / 30 \sim 1 / 4^{(16)}$ depth under the surface. However, the tendency is not found in the primary-recrystallized copper of the sample $A$, as shown in Fig. 12, indicating that the (200) X-ray intensity does not change at any depth under the surface.

\section{Discussion}

Most investigations of secondary recrystallization have recently focused on silicon steel sheets in relation to primary-recrystallized texture. Inokuti ${ }^{(15)}$ found that the primary recrystallization texture at $1 / 10$ depth under the sheet surface showed (110)[001], (210)[001] and (310)[001], while that at $1 / 2$ depth showed

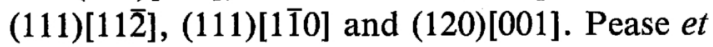
$a l .^{(16)}$ also observed that most of secondary grains nucleated in a certain depth range 10$80 \mu \mathrm{m}(1 / 30-1 / 4)$ below the sheet surface and also the texture gradient existed through the thickness. This discrepancy on the depth dependence may be due to different rolling schedule employed or different initial grain size before rolling. Another possible explanation is that inhibitors play an important role in the formation of potential nuclei for secondary recrystallization, as Inokuti ${ }^{(15)}$ pointed out. On the contrary, primary recrystallization texture of heavily cold rolled copper with fine grains showed only an extremely sharp cube orientation independent of depth from the sheet surface, as shown in Figs. 1 and 12. Nevertheless, the secondary recrystallization appeared abruptly during annealing. The preferred orientations of secondary grains in Figs. 2, 6, 10 and 11 are similar to those reported by Kronberg and Wilson ${ }^{(11)}$ and Sharp and Dunn ${ }^{(12)}$. It may be of interest to note that the distribution of the orientations of primary grains, excluding the cube orientation, overlap consistently those of the secondary grains, since the orientations of individual grains after primary recrystallization have never been observed so far. Namely, the distribution of the orientations of secondary grains in the fine grained sample $\mathrm{C}$ without ear, Fig. 6, corresponds approximately to that of primary grains etched preferentially, Fig. 5. Similarly, the primary grains with the orientation identical with secondary grains in Fig. 10 are found in the coarse grained sample $B$, as shown in Fig. 9. Furthermore, as shown in Fig. 3, some of primary grains in the ear of the fine grained sample A are quite similar in orientation to secondary grains in the sample A with ear, Fig. 2. These observations suggest that the potential nuclei of secondary recrystallization already exist in primary grains with an extremely sharp cube orientation and possess special orientation relationships to the cube orientation.

Many investigators showed that the orientations of secondary grains growing in a cube texture matrix were related to those of the primary grains by rotations about [111] and $[100]^{(9)-(12)}$, suggesting that the orientation relationships might be interpreted in terms of the Coincidence Site Lattice (C.S.L.) model. Let us discuss the growth mechanism of potential nuclei for secondary recrystallization on the basis of the C.S.L. model. It is recognized that the orientation relationships of two cubic lattices produced by rotation about [111] axis by $38^{\circ}$ (0.66 rad), $28^{\circ}(0.48 \mathrm{rad})$ and $22^{\circ}(0.38$ rad) are expressed as $\Sigma 7, \Sigma 13 \mathrm{~b}$ and $\Sigma 21 \mathrm{a}$, respectively. According to Brandon ${ }^{(22)}$, the maximum allowable deviation that can be accommodated in a nearest coincidence boundary, $\Delta \theta$, is given by $\Delta \theta=15^{\circ} \times(\Sigma)^{-1 / 2}$. Following his equation, $\Delta \theta$ is equal to $5.6^{\circ}(0.099 \mathrm{rad})$, $4.1^{\circ}(0.073 \mathrm{rad})$ and $3.2^{\circ}(0.057 \mathrm{rad})$ for $\Sigma 7$, $\Sigma 13 \mathrm{~b}$ and $\Sigma 21 \mathrm{a}$, respectively. Since the present experiment shows that the deviations of the sharp cube oriented grains from the ideal cube orientation are within $6^{\circ}(0.10 \mathrm{rad})$, circles with radii of $(\Delta \theta+6)^{\circ}$ are drawn for $\Sigma 7, \Sigma 13 b$ and $\Sigma 21 \mathrm{a}$ in Figs. 2, 3, 9 and 10. Most of the orientations of secondary grains in Figs. 2 and 10 fall within the circles.

It is also recognized that the rotation of two cubic lattices produced by rotation about [100] 
axis by $23^{\circ}(0.39 \mathrm{rad}), 19^{\circ}(0.32 \mathrm{rad})$ or $16^{\circ}$ $(0.28 \mathrm{rad})$ is expressed as $\Sigma 13 \mathrm{a}, \Sigma 37 \mathrm{a}$ or $\Sigma 25 \mathrm{a}$, respectively. $\Delta \theta$ of $\Sigma 13 \mathrm{a}, \Sigma 37 \mathrm{a}$ or $\Sigma 25 \mathrm{a}$ is $4.1^{\circ}$ $(0.072 \mathrm{rad}), 2.4^{\circ}(0.043 \mathrm{rad})$ or $3.0^{\circ}(0.052 \mathrm{rad})$, respectively. Circles with radii of $(\Delta \theta+6)^{\circ}$ for $\Sigma 13 \mathrm{a}, \Sigma 37 \mathrm{a}$ and $\Sigma 25 \mathrm{a}$ are also drawn in Figs. 2, $3,5,6,9$ and 11. Some of the orientations of secondary grains in Figs. 2, 6, and 11 are seen to be within the circles. Furthermore, the obtained results in Figs. 3, 5 and 9 can be explained approximately by using the orientation relationships of $\Sigma 7, \Sigma 13 \mathrm{a}, \Sigma 13 \mathrm{~b}, \Sigma 21 \mathrm{a}, \Sigma 25 \mathrm{a}$ and ¿37a.

In order to confirm experimentally the existence of potential nuclei with the C.S.L. boundaries in primary grains, SEM-ECP studies on the character of grain boundaries around grains with the off-cube orientation were carried out. An example is shown in Fig. 13, indicating SEM image and boundary character in primary grains of the sample C. A central grain in Fig. 13 is deeply etched in preference to surrounding grains. The orientation of the grain was found to deviate by $24^{\circ}$ $(0.41 \mathrm{rad})$ from the ideal cube orientation, in which case we ascertained the presence of the $\Sigma 13$ a boundary described above, as illustrated in Fig. 13. In a similar manner, some of the above mentioned C.S.L. boundaries were actually observed in the primary grains of the

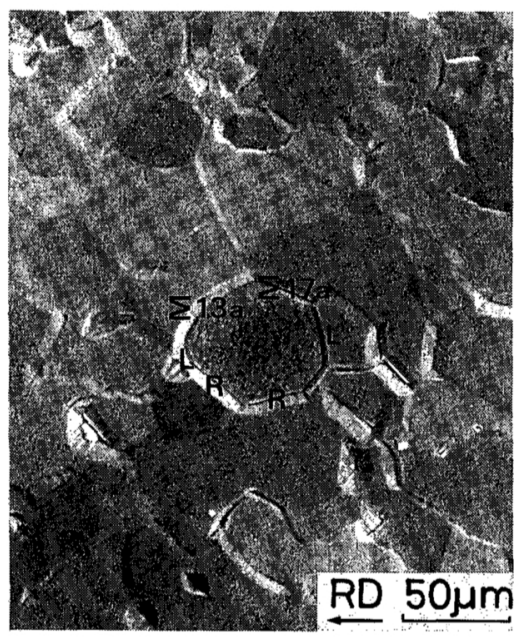

Fig. 13 Scanning electron micrograph and grain boundary structures after zigzag-rolled by $99 \%$ and annealed for $7.2 \mathrm{ks}$ at $1123 \mathrm{~K}$ in a vacuum of $2 \mathrm{~Pa}$. L: Low angle boundary ( $\Sigma 1)$. R: Random boundary $(\Sigma>29)$. samples B and C. Therefore, these grains seem to have potency to grow into secondary grains during annealing at higher temperatures. In spite of numerous experiments, we could not find any potential nuclei in the sample A, suggesting that the number of potential nuclei was too small.

All of the current analysis of nucleation of secondary grains take into account a size advantage of a potential nucleus ${ }^{(14)(15)(20)}$ and driving force resulting from grain boundary energies $^{(20)}$. However, the off-cube oriented grains with the C.S.L. boundary are as large as the adjacent grains, as shown in Fig. 13. The rate of secondary recrystallization in copper was experimentally uncontrollable. That is, secondary recrystallization in the sample $A$ without ear in a vacuum of $2 \times 10^{-2} \mathrm{~Pa}$ was not detected at $948 \mathrm{~K}$, while it proceeded too rapidly to control at $973 \mathrm{~K}$. Therefore, the potential nuclei are considered to grow just before secondary recrystallization. This implies that the size advantage of a potential nucleus does not hold in secondary recrystallization in copper. Pease et $a l .^{(16)}$ and Harase and Shimizu ${ }^{(17)(23)}$ have found that Goss-oriented grains in silicon steel sheets do not have sufficient size advantage to account for their selective growth as secondaries. Instead, Harase and Shimizu ${ }^{(23)}$ showed that the presence of grains with the C.S.L. boundaries was required as potential nuclei for secondary recrystallization. The present result is in good agreement with their conclusion. In addition, it should be noted that the above described C.S.L. boundaries in copper are considered to have higher grain boundary energies than other boundaries, since most of the primary grains in a sharp cube texture matrix consist of low angle boundaries. In other words, this means that the existence of a sharp cube texture, which suppresses normal grain growth, is required for secondary recrystallization in copper. Namely, the sharp cube texture acts as a kind of inhibitors to normal grain growth. This criterion is supported by the present observations that secondary recrystallization occurs when most of primary grains (the samples A and C) or clusters of primary grains (the sample B) have a sharp cube texture. 
The distribution of orientations of secondary grains in the sample A (Fig. 11) is quite different from that in the sample B (Fig. 10), although they are similar to those in the sample $\mathrm{C}$ (Fig. 6). Orientations of a few grains in Fig. 6, which can not be explained by the circles of the C.S.L. orientations, may result from slightly different rolling procedure. From the fact that the distribution of orientations of secondary grains in the sample $\mathrm{A}$ is similar in appearance to the sample $\mathrm{C}$ and is close to that of primary grains with the off-cube orientation in the sample C (Fig. 5), it is most likely that the obtained orientation relationship by rotation about [100] is applicable to fine grained samples. On the contrary, the orientation relationship by the rotation about [111] seems to appear in the coarse grained sample. The reason why the orientation axis depends on initial grain size, i.e. the degree of sharpness of primary cube texture, is uncertain at present, but it may be responsible for different deformation microstructure. As shown in Fig. 2, the orientation relationships with the rotation axes [111] and [100] coexist in the fine grained sample A with ear, suggesting that deformation microstructure in the ear is different from that in the center of the sheet. Thus, it is concluded that initial grain size as well as rolling procedure is a very important factor controlling the orientation relationship between secondary grains and the cube orientation, although many investigators did not take into account.

\section{Conclusion}

The primary and the secondary recrystallization texture have been examined in commercial copper with fine grains $(0.05 \mathrm{~mm})$ after straight-rolling and zigzag-rolling and with coarse grains $(0.5 \mathrm{~mm})$ after straight-rolling. The main results are summarized as follows.

(1) The primary recrystallization textures after straight-rolling and zigzag-rolling showed very sharp cube orientation. In the coarse grained sample, the primary recrystallization texture showed a weak cube mixed with random orientation according to X-ray diffraction.

(2) Primary recrystallization microstruc- tures were investigated by SEM-ECP in the fine grained sample after straight-rolling and zigzag-rolling. About 150 grains in the fine grained sample after straight-rolling had the orientations within $6^{\circ}(0.10 \mathrm{rad})$ from cube, while the grains about $10^{\circ}(0.174 \mathrm{rad})$ away from the cube orientation were present at the rate less than $1 \%$ in the sample after zigzagrolling. Clusters of grains with cube and scattered orientations were observed in the coarse grained sample after straight-rolling.

(3) Secondary recrystallization was affected by the initial grain size before cold rolling, rolling procedure, annealing conditions and the ear produced by heavy rolling reduction. The secondary recrystallization orientations are related to the primary recrystallization orientation; $\Sigma 7, \Sigma 13 \mathrm{a}, \Sigma 13 \mathrm{~b}, \Sigma 21 \mathrm{a}, \Sigma 25 \mathrm{a}$ and $\Sigma 37$. The orientation relationship by the rotation about [100] $(\Sigma 13 a, \Sigma 25 a, \Sigma 37 a)$ is applicable to fine grained sample and that by [111] $(\Sigma 7, \Sigma 13 b, \Sigma 21 a)$ is applicable to coarse grained sample. Some grains with such a C.S.L. boundary were found to be present in the primary-recrystallized sample, although such grains did not show any size advantage.

(4) The extremely sharp cube texture, consisting of low angle boundaries, inhibits the normal grain growth and leads to secondary recrystallization.

(5) Local inhomogeneities of primary recrystallization texture through the thickness of sheet were not observed in the straight-rolled copper.

\section{Acknowledgments}

The present authors would like to thank S. Watanabe for his experimental assistance. One of the authors (H. M) is also grateful to $\mathrm{H}$. Fukuda, Kawai Musical Instruments Mfg. Co., Ltd. for his kind encouragement and advice.

\section{REFERENCES}

(1) H. Takechi and K. Kawasaki: Shugo-soshiki (Texture), Ed. by S. Nagashima, Maruzen, (1984), p. 117.

(2) T. Kamijo, S. Shinya and H. Fukutomi: J. Japan Soc. Tech. Plasticity, 25 (1984), 375.

(3) A. A. Ridha and W. B. Hutchinson: Acta Met., 30 (1982), 1929.

(4) H. Hu: Texture, 1 (1974), 233. 
(5) H. Hu and S. R. Goodman: Trans. AIME, 227 (1963), 627.

(6) H. Makita, S. Hanada and O. Izumi: Acta Met. in press.

(7) H. Makita, S. Hanada and O. Izumi: J. Japan Inst. Metals, 50 (1986), 530.

(8) A. Okada, H. Mitsuji and H. Nakae: Trans. Japan Inst. Metals, 17 (1976), 497.

(9) O. Dahl and F. Pawlek: Z. Metallk., 28 (1936), 266.

(10) M. Cook and T. L. Richards: J. Inst. Metals, 66 (1940), 1.

(11) M. L. Kronberg and F. H. Wilson: Trans. AIME, 185 (1949), 501.

(12) M. Sharp and C. G. Dunn: J. Metals, 4 (1952), 42.

(13) H. Abe and S. Yamada: J. Japan Inst. Metals, 37 (1973), 1115.

(14) M. Matsuo, T. Sakai, M. Tanino, T. Shindo and S.
Hayami: Proc. 6th Int. Conf. on Texture of Materials, Ed, by S. Nagashima (1981), p. 918.

(15) Y. Inokuchi: Tetsu to Hagane, 70 (1984), 2033.

(16) N. C. Pease, D. W. Jones, M. H. L. Wise and W. B. Hutchinson: Met. Sci., 15 (1981). 203.

(17) J. Harase and R. Shimizu: Tetsu to Hagane, 72 (1986), s1350.

(18) J. E. May and D. Turnbull: Trans. AIME, 212 (1985), 769.

(19) M. Hillert: Acta Met., 13 (1965), 227.

(20) C. G. Dunn: Acta Met., 1 (1953), 163.

(21) M. Morita and K. Fukui: J. Japan Inst. Metals, 37 (1973), 861.

(22) D. G. Brandon: Acta Met., 14 (1966), 1479.

(23) J. Harase and R. Shimizu: Bull. Japan Inst. Metals, 23 (1986), 1009. 